\title{
Mineralization and Sticky Trickery of a Barnacle Cement Protein Resolved Computationally
}

\author{
AKSHITA KUMAR ${ }^{1}$, HARINI MOHANRAM ${ }^{1}$, CHANDRA S \\ VERMA $^{2}$ AND ALI MISEREZ ${ }^{1}$ \\ ${ }^{1}$ Nanyang Technological University \\ ${ }^{2}$ Bioinformatics Institute A*STAR \\ Presenting Author: akshita@ntu.edu.sg
}

Barnacles are dominant macrofoulers that adhere to a variety of immersed substrates through a protein-based cement. The sticky cement is a mixture of the five cement proteins (CP) that self-assemble into a permanently bonded layer. Among these proteins, Megabalanus rosa cement protein-20 (MrCP20) is a putative interfacial CP that conjoins barnacles' calcite shell to the adhesive cement. To probe its functional relevance in the barnacle cement, we investigate the molecular interactions between $\mathrm{MrCP} 20$ and calcium carbonate ions through molecular dynamics simulations. Our work highlights that MrCP20 sequesters free ions on its highly charged surface through a disorder-order interplay. The conformational landscape of MrCP20 illustrates similar ordering behaviour upon calcite surface adsorption. Detailed structural examinations indicate that the energetically favourable interactions with calcium carbonate (ions and calcite mineral surface) are mediated by $(i)$ charged functional groups that flank the structured regions of $\mathrm{MrCP} 20$ and (ii) networks of water molecules. In vitro experiments indicate that $\mathrm{MrCP} 20$ favours precipitation of a less stable polymorph of calcium carbonate (vaterite). This comprehensive study evidences the dual functional role of MrCP20: (a) regulation of mineralization in barnacle cement and (b) underwater adhesion to surfaces like calcite. 